\title{
EFEITO DE FONTES, DOSES EE GRANULOMETRIAS DE CALCÁRIOS \\ NA FIXAÇÃO DO N2 E NO ESTADO NUTRICIONAL DE CAUPI (1)
}

\section{JOSÉ PAULO VIEIRA DA COSTA(2) \& NEWTON PEREIRA STAMFORD(3)}

\section{RESUMO}

Foi conduzido um experimento em casa de vegetação em um solo Podzólico Vermelho Amarelo latossólico para verificar o efeito dos calcários Megaó, Magnecal e Cambucá, usados nas doses de 3,$0 ; 6,0$ e 9,0 t/ha com três granulometrias (comercial, 200 e 400 malhas/pol), na atividade das enzimas nitrogenase e redutase do nitrato e no estado nutricional de caupi (Vigna unguiculata - cv "Pitiúba"). Todos os tratamentos receberam inoculaçâo com a estirpe J-01, selecionada anteriormente. A atividade enzimática, bem, como a concentração do nitrogênio nas folhas, nas formas nítrica e amoniacal, não sofreram influências consideráveis da adição dos calcários. A absorção do nitrogênio, do fósforo, do cálcio e do magnésio aumentou com a calagem e os teores desses nutrientes foram maiores quando os tratamentos receberam o calcário com maior teor de magnésio. As fontes, as doses e as grānulometrias dos calcários não exerceram influência na acumulação do potássio pela planta.

Termos para indexação: calcário, caupi, fixação do $\mathrm{N}_{2}$

(1) Parte da Dissertaçăo de Mestrado do primeiro autor, apresentado à UFRPE. Aceito para publicaçăo em 21 de fevereiro de 1991.

(2) Prof. Assistente, Departamento de Agronomia - CECA/UFAL - 57.080 - Maceló - AL

(3) Prof. Adjunto, Departamento de Agronomia - UFRPE - 52071 - Recife PE 


\section{ABSTRACT}

\section{EFFECT OF SOURCES, LEVELS AND LIMES PARTICLES SIZE ON $\mathrm{N}_{2}$ FIXATION AND NUTRICIONAL STATUS.}

A greenhouse experiment was carried out to study the effects of regional limes on $\mathrm{N}_{2}$ fixation and cowpea nutricional status in a Red-Yellow Podzolic soil. Negaó, Magnecal and Cambucá limes were used in three crescent rates (3.0,6.0 and 9.0 t/ha) and different particle size (comercial, 200 mesh and 400 mesh). All treatments were inoculated with $\mathrm{J}-01$, a local Bradyrhizobium strain. Enzime activity as well as $\mathrm{N}-\mathrm{NH}_{4}$ and $\mathrm{N}-\mathrm{NO}_{3}$ concentration on leaves were not infouenced by lime addition. Nitrogen, phosphorus, calcium and magnesium accumulated by cowpea leaves increased with liming and highest concentration were obtained with cambuca lime that has more magnesium content. Potassium accumulation was not influenced by sources, rates and particles size.

Index terms: lime, cowpea, $\mathrm{N}_{2}$ fixation.

\section{INTRODUÇÃO}

A fixação simbiótica do nitrogênio, o processo de nodulação e o rendimento das culturas estão condicionados aos efeitos da acidez do solo.

Os resultados de aumentos na produção das culturas obtidos pelo emprego de calcário são os mais diversos possíveis, talvez devido à sua granulometria, ao valor de neutralização da acidez ou até à metodologia para cálculo da dose a ser usada (RIOS, 1968).

O emprego de calcário no Brasil ainda é muito limitado, embora - seu uso seja um dos mais importantes fatores do aumento da produtividade. Isto se deve ao desconhecimento dos benefícios dessa prática $e$, em parte, às idéias alarmistas sobre os perigos de excesso de calagem em solos tropicais, divulgadas nos anos passados e que persistem até hoje (RAIJ, 1980).

PURCINO et alii (1981) desenvolveram estudos num Latossolo Vermelho Escuro do Brasil Central com duas cultivares de feijão alado para verificarem crescimento e parâmetros nudulares e encontraram efeitos positivos da adição do cálcio ao solo na fixação do nitrogênios nas plantas.

Testando vinte e uma estirpes de Bradyrhizobium quanto à eficiência em nudular três cultivares de caupi, KEYSER \& MUNNS (1979) observaram que as estirpes do grupo caupi apresentaram grande variação quanto à tolerância a acidez. 
SCHOLES et alii (1981), trabalhando com cinco leguminosas, verificaram que a inoculação com Bradyrhizobium foi fundamental para a obtenção de altos níveis de nitrogênio fixado apenas para uma espécie, o mesmo não sendo observado para aplicação de calcário que foi eficiente para a maioria das leguminosas estudadas.

FRANÇA et alii (1973) estudaram os efeitos de magnésio, micronutrientes e calagem em soja. A calagem aumentou significativamente os teores de nitrogênio, de fósforo, de cálcio e de magnésio na parte aérea. Apenas a absorção de potássio não aumentou com a calagem.

Parece que não existe correlação entre $0 \mathrm{pH}$ e os processos simultâneos de absorção do amônio e redução do acetileno. A influência do $\mathrm{pH}$ na absorção do amônio é contraditória: ora aumenta, ora diminui levemente com o aumento da acidez. Isso pode resultar de interações de idade da planta, espécie da planta, temperatura, ânion acompanhante na absorção do amônio (MARCUS-WINER \& RAINS, 1982).

O objetivo deste trabalho foi obter informações no que concerne à aplicação de diferentes fontes, doses e granulometrias de calcário na fixação do $\mathrm{N}_{2}$ e no estado nutricional de caupi.

\section{MATERIAL E MÉTODOS}

O experimento foi conduzido em casa de vegetação, utilizando-se amostras de um solo Podzólico Vermelho Amarelo latossólico (BRASIL, 1973), coletado à profundidade de $20 \mathrm{~cm}$ no município de Goiana-PE, e cuja análise química foi a seguínte: $\mathrm{pH}$ em $\left(\mathrm{H}_{2} \mathrm{O}(1: 2,5)=4,5 ; \mathrm{Al}^{+}+(\mathrm{KCl} 1 \mathrm{~N})=0,5 \mathrm{meq} / 100 \mathrm{ml}\right.$ de solo; $\mathrm{Ca}^{2+}+$ $\mathrm{Mg}^{2++}(\mathrm{KLC1} 1 \mathrm{~N})=2,2 \mathrm{meq} / 100 \mathrm{ml}$ de solo; $\mathrm{P}($ Mehlich -1$)=5 \mathrm{ppm}$ e $K($ Mehlich -1$)=5 \mathrm{ppm}$. No trabalho, foram utilizados os calcários Megaó, Magnecal e Cambucá nas doses de 3,0; 6,0 e 9,0t/ha, usando como base o equivalente em $\mathrm{CaCO}_{3}$. Para avaliar o efeito da granulometria de cada fonte, foram usados os graus de finura: 0 primeiro tal como o calcário se apresenta na sua forma comercial e os dois outros produzidos em laboratório, sendo um com partículas menores que 200 malhas/pol e o outro com partículas menores que 400 malhas/pol. A localização das jazidas e as características dos calcários encontram-se no Quadro 1. Os tratamentos foram dispostos num arranjo fatorial $3 \times 3 \times 3$ (três fontes, três doses e três granulometrias de calcário), distribuídos em delineamento inteiramente ao acaso com três repetições.

A cultivar de caupi escolhida foi Pitiúba, tendo em vista que em ensaio preliminar a mesma comportou-se como a mais indicada, e também por ser uma das mais usadas na região. 
QUADRO 1 - Caracterização dos calcários

\begin{tabular}{|c|c|c|c|c|c|c|c|c|}
\hline \multirow[t]{2}{*}{ CALCARIO } & \multicolumn{3}{|c|}{$\begin{array}{r}\text { LOCALIZAC } A 0 \\
\text { JAZIDA }\end{array}$} & DA & $\mathrm{CaO}$ & $\mathrm{MgO}$ & $\mathrm{PN}$ & PRNT \\
\hline & & & & & $-\%-$ & $-\%-$ & $-\%-$ & - \% \\
\hline MEGAO & & Suru & & & 42,3 & 7,4 & 93,79 & 93,53 \\
\hline MAGNECAL & & Paul & $\mathrm{ta}$ & & 30,0 & 11,0 & 80,90 & 45,81 \\
\hline CAMBUCA & Sta & Maria & $\mathrm{Ca}$ & bucà & 34,4 & 17,8 & 105,73 & 75,28 \\
\hline
\end{tabular}

Todos os vasos receberam inoculação com Bradyrhizobium (estirpe J-01), isolada de caupi cultivado na Zona da Mata de Pernambuco.

Os calcários foram misturados com o solo, procurando-se promover a mais completa homogeneização.

$A$ adubação básica foi efetuada nas doses equivalentes a 30 $\mathrm{Kg} / \mathrm{ha}$ de $\mathrm{N} ; 150 \mathrm{Kg} / \mathrm{ha}$ de $\mathrm{P}_{2} 0_{5}$ e $200 \mathrm{Kg} / \mathrm{ha}$ de $\mathrm{K}_{2} \quad 0$. A adubação com micronutrientes foi feita através da adição de $1 \mathrm{ml} / \mathrm{Kg}$ de solo de uma solução contendo por litro: $15,8 \mathrm{~g} \mathrm{e} \mathrm{CuSO}_{4} ; 20,0 \mathrm{~g}$ de $\mathrm{FeSO}_{4} .7 \mathrm{H}_{2} \mathrm{O} ; 8,9 \mathrm{~g}$ de $\mathrm{ZnSO}_{4} .7 \mathrm{H}_{2} \mathrm{O} ; 1,0 \mathrm{~g}$ de $\mathrm{H}_{3} \mathrm{BO}_{3} ; 0,79$ de $\mathrm{NaMoO}_{4}$ e 20,0g de ácido cítrico.

Foram plantadas cinco sementes por vaso e, após a germinação, procedeu-se ao desbaste deixando-se duas plantas por vaso.

A atividade da redutase do nitrato foi determinada quarenta e quatro dias após o plantio, através da determinação do nitrito produzido após a incubação. Foi usado o método de colorimetria por reação com n-naftil etileno diamina e sulfanilamida ("kill Jwice").

Das mesmas folhas utilizadas na amostragem para determinação da atividade da redutase do nitrato, pesou-se um grama para o fracionamento do nitrogênio, compreendendo as determinaçōes de $\mathrm{N}_{-} \mathrm{NH}_{4}^{+}, \mathrm{N}-\mathrm{NO} \frac{-}{3}$ e $\mathrm{N}$-protéico. Com a finalidade de paralisar a atividade enzimática, as amostras foram colocadas em $20 \mathrm{ml}$ de etanol a $80 \%$. Foram determinados amônio e nitrato pelo método de arraste a vapor descrito por BREMNER \& KEENEY (1965) e nitrogênio protéico, no resíduo, após secagem a $65^{\circ} \mathrm{C}$, como descrito por BREMNER (1965).

Quarenta e cinco dias após o plantio, realizou-se a colheita, separando-se o sistema radicular da parte aérea. O sistema radicular foi colocado em recipiente hermeticamente fechado. Fez-se a injeção de acetileno, correspondendo a $10 \%$ do volume do recipiente. Uma hora após, foi avaliada a atividade da nitrogenase, medindo-se, por cromatografia gasosa, a quantidade de etileno evoluido.

O tecido vegetal da parte aérea foi moido, passando por peneira $n^{\circ} 20$, e em seguida feita a digestāo nitro-perclórica da maneira descrita por SARRUGE \& HAAG 1974 ; para determinação dos nu- 
trientes fósforo, potássio, cálcio e magnésio, e usou-se a digestão sulfúrica para determinação do nitrogênio.

A determinaçāo do fósforo foi efetuada por colorimetria, a do potássio por fotometria de chama e a do cálcio e do magnésio por espectrofotometria de absorção atómica.

O nitrogênio total na planta foi determinado pelo método de Kjeldhal, de acordo com o procedimento descrito por BREMNER (1965).

\section{RESULTADOS E DISCUSSÃO}

As atividades total e relativa da nitrogenase não foram influenciadas pelas fontes de caicário utilizadas, conforme pode ser observado pelos dados do Quadro 2. Uma possível explicaçāo para isto pode ser a sustentada por MORRIS(1967) de que a inexistência de resposta à calagem pelas leguminosas tropicais é devida à extrema capacidade que essas plantas possuem de extrair o cálcio necessário ao seu crescimento, entretanto FRANCO \& DAY (1980) encontraram efeito positivo da calagem na atividade da nitrogenase em solo Podzólico Vermelho Amarelo.

Com relação à influência da granulometria na atividade total da nitrogenase (Quadro 6), verificou-se efeito signifirativo, particularmente para o calcário cambucá, o qual apresentou maior atividade na granulometria menor que 400 malhas/pol (Quadro 2).

A atividade da redutase do nitrato não sofreu efeito das fontes, doses e granulometrias dos calcários (Quadros 2 e 6). Os dados sugerem que provavelmente a época para avaliação da atividade dessa enzima em caupi é diferente da utilizada neste trabalho.

O efeito da calagem nos niveis de amónio e de nitrato na parte aérea não foi significativo. Os dados referentes ao nitrogênio protéico na parte (Quadro 3) mostraram efeitos significativos das fontes, das doses e das granulometrias, sendo as doses mais elevadas e a granulometria mais fina nos calcários megaó e magnecal os que promoveram melhores resultados. Dados coerentes com relação aos efeitos da calagem nos teores de nitrogênio protéico foram obtidos por SANTOS (1982), trabalhando com Vigna unguiculata.

O nitrogênio total acumulado na parte aérea (Quadro 4) seguiu a mesma tendência observada para o nitrogênio protéico. A ausência de efeito para amonio e nitrato parece realmente encontrar justificativano fato de que a maior parte do nitrogênio havia sido meta. bolizado. 
QUADRO 2 - Efeitos de fontes, niveis e granulometrias dos calcários na atividade das enzimas nitrogenase e redutase do nitrato. (1)

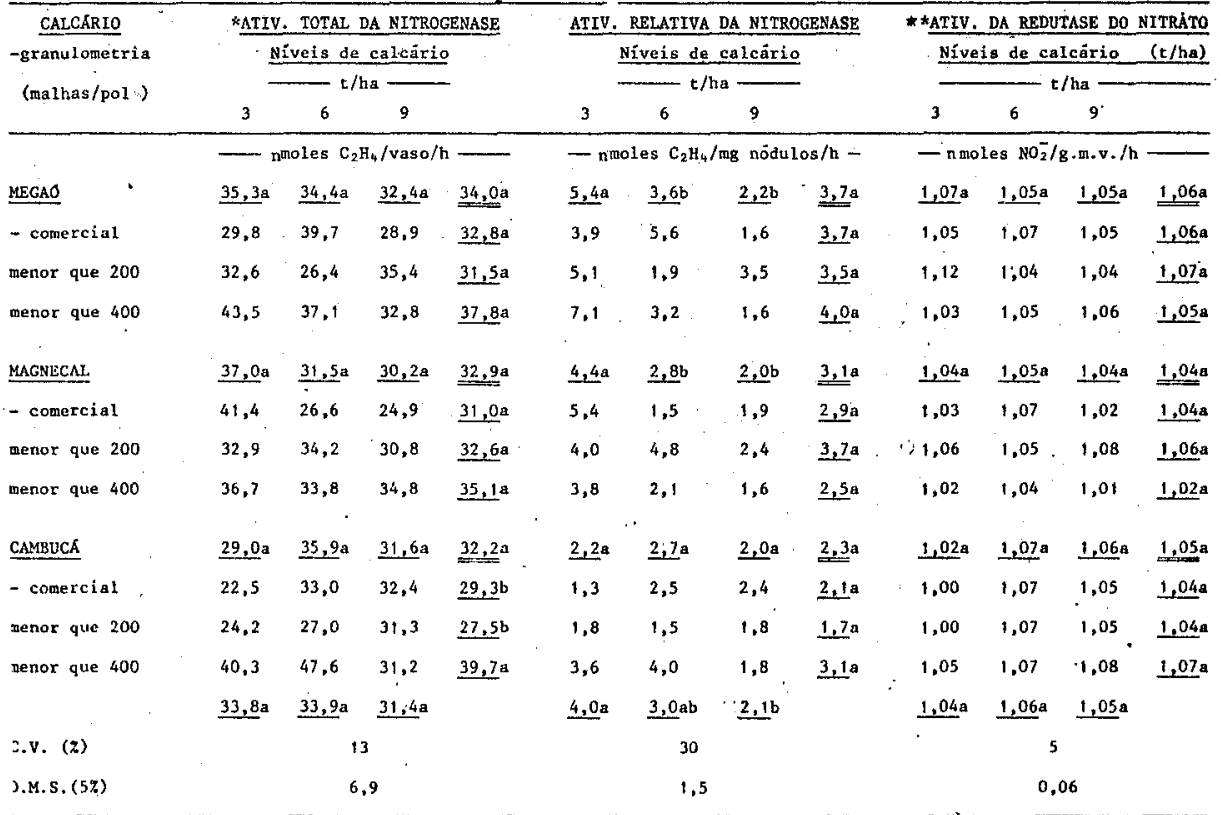

(1) Para fontes, niveis e granulometrias, os números com a mesma letra náo diferem entre sí peloteste de Tukey a $5 \%$.

* Dados transformados em $\sqrt{x}$

** Dados transformados em $\sqrt{x+1}$ 
QUADRO 3 - Efeitos de fontes, niveis e granulometrias dos calcários na quantidade de amónio, nitrato e nitrogénio protéico da parte aérea. (1)

\begin{tabular}{|c|c|c|c|c|c|c|c|c|c|c|c|c|}
\hline \multirow{3}{*}{$\begin{array}{l}\text { CALCARIO } \\
\text {-granulometria } \\
\text { (malhas/pol) }\end{array}$} & \multicolumn{2}{|c|}{ AMONIO } & \multicolumn{2}{|c|}{ PLANTA } & \multicolumn{2}{|c|}{ NITRATO } & \multicolumn{2}{|c|}{ PLANTA } & \multicolumn{4}{|c|}{ NITROSENIO PROTEICO NA PLANTA } \\
\hline & \multicolumn{4}{|c|}{ Niveis de cnlcärio (t/ha) } & \multicolumn{4}{|c|}{ Níveis de calcärio $(t / h a)$} & \multicolumn{4}{|c|}{ Niveis de calcäio ( $\mathrm{t} / \mathrm{ha}$ ) } \\
\hline & 3 & 6 & 9 & & 3 & 6 & 9 & & 3 & 6 & 9 & \\
\hline \multirow[b]{2}{*}{ MEGAO } & \multicolumn{4}{|c|}{ — nmoles $\mathrm{NH}_{4}^{+} / \mathrm{g}, \mathrm{m}, \mathrm{v}$. } & \multicolumn{4}{|c|}{ - nmoles $\mathrm{N}_{3}^{-} / \mathrm{g} \cdot \mathrm{m} . \mathrm{v}}$. & \multicolumn{3}{|c|}{ - mg/vaso } &  \\
\hline & $33.1 \mathrm{a}$ & $\underline{38,1 \mathrm{a}}$ & $37,2 \mathrm{a}$ & $36,1 \mathrm{a}$ & $\underline{38,2 a}$ & $\underline{38,7 \mathrm{a}}$ & $37,4 \mathrm{a}$ & 38,18 & $165 b$ & $\underline{161 \mathrm{~b}}$ & $\underline{209 a}$ & $178 \mathrm{~b}$ \\
\hline - comercial & 35,4 & 39,4 & 36,8 & $37,2 \mathrm{a}$ & 38,0 & 38,2 & 43,9 & $40,0 \mathbf{a}$ & 110 & 155 & 195 & $153 \mathrm{~b}$ \\
\hline menor que 200 & 32,6 & 38,4 & 40,8 & $37,3 a$ & 35,2 & 37,0 & 33,0 & $35,1 a$ & 142 & 148 & 243 & $178 \mathrm{ab}$ \\
\hline mener & 31.4 & 36,6 & 34,1 & $34,0 \mathrm{n}$ & 41,4 & 41,0 & 35,3 & $39,2 n$ & 242 & 18D & 190 & $204 \mathrm{n}$ \\
\hline MAGNECAL & $\underline{35,7 a}$ & $\underline{35,9 a}$ & $39,3 \mathrm{a}$ & $37,0 \mathrm{a}$ & $\underline{41,3 a}$ & $39,0 \mathrm{a}$ & $40,1 \mathrm{a}$ & $40,1 \mathrm{a}$ & $159 \mathrm{~b}$ & $\underline{173 b}$ & $218 a$ & $184 b$ \\
\hline - comercial & 41,0 & 40,6 & 39,8 & $40,5 \mathrm{a}$ & 40,4 & 39,2 & 39,3 & $39,6 \mathrm{a}$ & 162 & 170 & 162 & $165 b$ \\
\hline menor que 200 & 29,0 & 36,6 & 35,8 & $33,8 \mathrm{a}$ & 38,6 & 36,9 . & $.36,8$ & $37,4 \mathrm{a}$ & 158 & 147 & 195 & $167 \mathrm{~b}$ \\
\hline menor que 400 & 37,0 & 30,4 & 42,4 & $36,6 \mathrm{a}$ & 44,8 & 40,9 & 44,2 & $43,3 a$ & 158 & 202 & 297 & $219 \mathrm{a}$ \\
\hline CAMBUCA & $34,4 a$ & $37,3 \mathrm{a}$ & $31,8 \mathrm{a}$ & $34,5 \mathrm{a}$ & $35,8 \mathrm{a}$ & $38,4 a$ & $31,9 a$ & $35,4 a$ & $220 \mathrm{a}$ & $232 \mathrm{a}$ & $\underline{232 \mathrm{a}}$ & $228 \mathrm{a}$ \\
\hline - comercial & 32,9 & 36,8 & 27,3 & $32,3 a$ & 39,8 & 40,3 & 28,8 & $36,3 \mathrm{a}$ & 188 & 285 & 222 & $232 a$ \\
\hline menor que 200 & 35,4 & 35,3 & 32,4 & $34,4 \mathrm{a}$ & 39,4 & 38.0 & 34,2 & $37,2 a$ & $210^{\circ}$ & 173 & 262 & $\underline{215} \mathrm{a}$ \\
\hline menor que 400 & 34,8 & 39,7 & 35,6 & $36,7 \mathrm{a}$ & 28,2 & 36,9 & 32,8 & $32,6 \mathrm{a}$ & 262 & 238 & 213 & $238 \mathrm{a}$ \\
\hline . & $34,4 \mathrm{a}$ & $37,1 \mathrm{a}$ & $36,1 \mathrm{a}$ & & $38,4 a$. & $38,7 \mathrm{a}$ & $36,5 a$ & & $181 \mathrm{a}$ & $189 \mathrm{a}$ & $\underline{2204}$ & \\
\hline c.v. (z) & & & & & & & & & & & & \\
\hline D.M.S. (5\%) & & & & & & & & & . & & & \\
\hline
\end{tabular}

(1) Para fontes, niveis e granulometrias, os números com a mesma letra năo diferem entre si pelo teste de Tukey a $5 \%$. 


\begin{tabular}{|c|c|c|c|c|c|c|c|c|c|c|c|c|}
\hline \multirow{2}{*}{$\begin{array}{c}\text { CALCARYO } \\
\text {-granulometria } \\
\text { (malhas/pol) }\end{array}$} & \multicolumn{4}{|c|}{ N TOTAL NA PARTE ARREA } & \multicolumn{4}{|c|}{ P TOTAL NA PARTE AERE: } & \multicolumn{4}{|c|}{$X$ TOTAL SA PARTE AEREA } \\
\hline & 3 & 6 & 9 & & 3 & 6 & 9 & & 3 & 6 & 9 & \\
\hline & \multicolumn{4}{|c|}{$\mathrm{mg} / \mathrm{vaBO}$} & \multicolumn{4}{|c|}{$\left[\mathrm{mg} / \mathrm{vaBO} \mathrm{O}^{-}\right.$} & \multicolumn{4}{|c|}{${ }^{-m g / v a B o}-$} \\
\hline MECAO & 373a & 382a & $402 \mathrm{a}$ & $386 \mathrm{~b}$ & 35a & $35 \mathrm{a}$ & 36a & $35 \mathrm{~b}$ & $118 \mathbf{a}$ & $116 \mathrm{a}$ & $131 \mathrm{a}$ & 122. \\
\hline - comercial & 331 & 332 & 374 & $346 \mathrm{~b}$ & 30 & 31 & 28 & 30b & 112 & 114 & 113 & 113a \\
\hline menor que 200 & 379 & 378 & 401 & $\underline{386 \mathrm{ab}}$ & 30 & 36 & 41 & $\underline{36 \mathrm{ab}}$ & 118 & 116. & 196 & $123 \mathrm{a}$ \\
\hline menor que 400 & $409^{\circ}$ & 436 & 431 & 425a & 46 & 38 & 38 & $41 \mathrm{a}$ & 125 & 119 & 144 & 129. \\
\hline MAGNECAL & $396 \mathrm{a}$ & 423a & $463 a$ & $427 a b$ & $\underline{29 b}$ & 39ab & 42a & $\stackrel{37 \mathrm{ab}}{\mathrm{m}}$ & $124 \mathrm{a}$ & $120 \mathrm{a}$ & $116 \mathrm{a}$ & $120 \mathrm{a}$ \\
\hline - comercial & 333 & 381 & 402 & $372 \mathrm{~b}$ & 27 & 30 & 44 & $34 \mathrm{a}$ & 104 & 119 & 100 & $108 \mathrm{a}$ \\
\hline menor que 200 & 405 & 422 & 482 & $435 \mathrm{ab}$ & 32 & 39 & 41 & 37a & 130 & 115 & 117 & $121 \mathrm{a}$ \\
\hline waior que 400 & 450 & 465 & 506 & $474 \mathrm{a}$ & 28 & 49 & .40 & $\underline{39} \mathrm{a}$ & 138 & 126 & 131 & 132a \\
\hline CAMBUCA & 415n & $477 \mathrm{a}$ & $480 a$ & $457 \mathrm{a}$ & 42a & 43a & $\underline{52 a}$ & 465a & III. & 120. & 1258 & $119 \mathrm{~m}$ \\
\hline - comercial & 371 & 461 & 450 & 427a & 39 & 37 & 49 & 42 $\mathrm{a}$ & 102 & 100 & 122 & $108 \mathrm{a}$ \\
\hline menor que 200 & 425 & 475 & 486 & $462 \mathrm{a}$ & 42 & 44 & 51 & $46 a$ & 117 & 137 & 133 & 129a \\
\hline menor que 400 & 448 & 496 & 505 & $483^{\circ}$ & 46 & 48 & 55. & 50 a & 115 & 122 & 119 & $119 a^{\circ}$ \\
\hline & $\underline{395 a}$ & 427a & $448 \mathrm{a}$ & & $35 \mathbf{a}$ & $\underline{39 a}$ & $43 a$ & & $\underline{118 a}$ & $119 a$ & $\underline{124 a}$ & \\
\hline c.v. (z) & & ic & & & & & & & . & 1 & & \\
\hline D.M.S. (5z) & & 75 & & & & & & & & 3 & & \\
\hline
\end{tabular}

(1) Para fontes, nivels $\theta$ granulometrias, os números com a mesma letra năo diferem entre si pelo teste de Tukey a $5 \%$. 
Para o fósforo, observou-se que os calcários magnecal e cambucá apresentaram comportamento semelhante e estatisticamente superior ao megaó (Quadro 4). O calcário cambucá mostrou resultados melhores que o megá, indicando a influência do magnésio na absorção do fóstoro. Efeitos positivos da calagem na absorção desse nutriente também foram encontrados por JONES \& FREITAS (1970) em soja e LOVADINI et alii (1977) em leguminosas tropicais.

A absorção de potássio não foi influenciada pela calagem, como encontrado por STAMFORD et alii (1980).

A calagem, como era de se esperar, influenciou a absorção do cálcio e do magnésio pelas plantas (Quadro 5). O magnésio acumulado na parte aérea apresentou resposta mais pronunciada do que o cálcio, tanto para fotes como para doses e granulometrias, o que evidencia o efeito daquele nutriente (Quadros 5 e 6).

QUADRO 5 - Efeitos de fontes, niveis e granulometria dos calcários na quantidade de cálcio e de magnésio da parte aérea. (1)

\begin{tabular}{|c|c|c|c|c|c|c|c|c|}
\hline \multirow{3}{*}{$\begin{array}{l}\text { CALCARIO } \\
\text {-granulometria } \\
\text { (malhas/pol) }\end{array}$} & \multicolumn{4}{|c|}{ CÁLCIO TOTAL NA PARTE AEREA } & \multicolumn{4}{|c|}{ MAGNESIO TOTAL NA PARTE AEREA } \\
\hline & \multicolumn{4}{|c|}{ Niveis de calcário ( $t / h a)$} & \multicolumn{4}{|c|}{ Niveis de calcario (t/ha) } \\
\hline & 3 & 6 & \multicolumn{2}{|l|}{9} & 3 & 6 & \multicolumn{2}{|l|}{9} \\
\hline \multirow[b]{2}{*}{ MEGAO } & \multicolumn{4}{|c|}{$\longrightarrow \mathrm{mg} / \mathrm{vaso} \longrightarrow$} & \multicolumn{4}{|c|}{$\longrightarrow \mathrm{mg} / \mathrm{vaso} \ldots$} \\
\hline & $\underline{165 a}$ & $165 a$ & $174 a$ & $168 \mathrm{~b}$ & $\underline{63 b}$ & $\underline{68 \mathrm{~b}}$ & $82 \mathrm{a}$ & $71 \mathrm{~b}$ \\
\hline - comercial & 151 & 149 & 154 & $151 \mathrm{~b}$ & 56 & 62 & 71 & 63b \\
\hline menor que 200 & 151 & 154 & 179 & $161 \mathrm{ab}$ & 64 & 64 & 83 & $70 \mathrm{ab}$ \\
\hline menor que 400 & 194 & 192 & 190 & $192 \mathrm{a}$ & 70 & 78 & 91 & $80 \mathrm{a}$ \\
\hline MAGNECAL & $164 a$ & $154 a$ & $177 \mathrm{a}$ & $165 \mathrm{~b}$ & $\underline{65 \mathrm{~b}}$ & $79 a$ & $84 a$ & $\stackrel{76 \mathrm{~b}}{=}$ \\
\hline - comercial & 137 & 142 & 158 & $146 \mathrm{~b}$ & 48 & 67 & 72 & $\underline{62 b}$ \\
\hline menor que 200 & 177 & 139 & 173 & $163 \mathrm{ab}$ & 72 & 76 & 84 & $77 a$ \\
\hline menor que 400 & 178 & 182 & 199 & $186 \mathbf{a}$ & 74 & 93 & 95 & $\underline{87 a}$ \\
\hline CAMBUUCE & $174 \mathrm{~b}$ & $209 a$ & $220 a$ & $201 a$ & $70 \mathrm{~b}$ & $91 \mathrm{a}$ & $95 a$ & $85 a$ \\
\hline - conercial & 148 & 151 & 192 & $164 \mathrm{~b}$ & 66 & 83 & 82 & $77 \mathrm{~b}$ \\
\hline menor que 200 & 180 & 241 & 241 & $\underline{221 a}$ & 69 & 89 & 96 & $85 a b$ \\
\hline \multirow[t]{2}{*}{ menor que 400} & 194 & 234 & 226 & $218 a$ & 76 & 101 & 107 & $95 a$ \\
\hline & $168 \mathbf{a}$ & $176 \mathbf{a}$ & $190 a$ & & $66 \mathrm{~b}$ & $79 \mathrm{a}$ & $87 a$ & \\
\hline C.V. (\%) & \multicolumn{4}{|c|}{10} & \multicolumn{2}{|c|}{8} & & \\
\hline D.M.S. (5) & \multicolumn{2}{|c|}{31} & & & \multicolumn{2}{|c|}{10} & & \\
\hline
\end{tabular}

(1) Para fontes, niveis e granulometrias, os números com a mesma letra não diferem entre si pelo teste de Tukey a $5 \%$. 
QUADRO 6 - Efeitos das granulometrias nos parâmetros da planta (1).

\begin{tabular}{|c|c|c|c|c|c|c|c|c|c|c|c|c|c|c|c|c|c|}
\hline \multirow{3}{*}{$\begin{array}{l}\text { GRANULOMETRIA } \\
\text { (malhas/pol-) }\end{array}$} & \multirow{3}{*}{$\begin{array}{l}\text { Nivel de } \\
\text { calcärio } \\
\text { (c/ha) }\end{array}$} & \multicolumn{3}{|c|}{ NODULACAO } & \multicolumn{3}{|c|}{ ATIVIDADE ENZTMATIGA } & \multicolumn{5}{|c|}{ ABSORGAO DE NUTRIENTES } & \multicolumn{3}{|c|}{ FRAGIONAMENTO } & \multicolumn{2}{|c|}{ RENDIMENTO } \\
\hline & & \multirow[b]{2}{*}{ No } & \multirow{2}{*}{$\frac{\text { Peso }}{\text { total }}$} & \multirow{2}{*}{$\frac{\text { Peso }}{\text { médio }}$} & \multicolumn{2}{|c|}{ Nitrogenase } & \multirow[t]{2}{*}{ ARN 3/ } & \multirow{2}{*}{$\mathbf{N}$} & \multirow{2}{*}{$\ddot{p}$} & \multirow[b]{2}{*}{ K } & \multirow[b]{2}{*}{$\mathrm{Ca}$} & \multirow[b]{2}{*}{$\mathrm{Mg}$} & \multirow[b]{2}{*}{$\mathrm{NH}_{4}^{+}$} & \multirow[b]{2}{*}{$\mathrm{NO}_{3}^{-}$} & \multirow[b]{2}{*}{ N-protēico } & \multirow[b]{2}{*}{ parte aërea } & \multirow[b]{2}{*}{ raiz } \\
\hline & & & & & total I/ & relativa $2 /$ & & & & & & & & & & & \\
\hline & & & $-\mathrm{mg} /$ & vaso - & & & & $\ldots$ & - & $\mathrm{mg} / \mathrm{vas}$ & 0 & {[} & -umoles & /g m.v. & $-\mathrm{mg} / \mathrm{vas} \mathrm{-}$ & $--g-$ & + \\
\hline \multirow[t]{5}{*}{ Comercial } & & $104 a$ & $\underline{397 \mathbf{a}}$ & $3,84 \mathrm{a}$ & $31,0 \mathbf{a}$ & $\underline{8,7} \mathbf{a}$ & $1,05 a$ & $\underline{382} \mathrm{~b}$ & $\underline{35} \mathbf{a}$ & $110 \mathrm{a}$ & $153 \mathrm{~b}$ & $\underline{68 b}$ & $36,6 \mathrm{a}$ & $38,6 a$ & $183 \mathbf{a}$ & $12,9 b$ & $2,14 a$ \\
\hline & 3 & 95 & 323 & 3,44 & 31,2 & 10,6 & 1,03 & 345. & 32 & 106 & 145 & 57 & 36,4 & 39,4 & 153 & 11,6 & 2,14 \\
\hline & 6 & 114 & 404 & 3,56 & 33,1 & 9,6 & 1,07 & 391 & 33 & 111 & 147 & $71:$ & 38,9 & 39,2 & 203 & 13,1 & 2,09 \\
\hline & 9 & 104 & 463 & 4,52 & 28,7 & 5,9 & 1,04 & 409 & 40 & 112 & 168 & 75 & 34.6 & 37,3 & 193 & 13,8 & 2,20 \\
\hline & & ' & & 、 & & · & & & & & & & & & & & \\
\hline \multirow[t]{4}{*}{ Menor que 200} & & $\underline{101 \mathrm{a}}$ & $406 a$ & $4,06 \mathrm{a}$ & $30,5 a$ & $\underline{8,9 a}$ & $1,06 a$ & $428 \mathrm{ab}$ & $40 \mathrm{a}$ & $125 a$ & $182 \mathrm{ab}$ & $77 \mathrm{ab}$ & $35,1 a$ & $35,4 \mathrm{a}$ & $186 a$ & $14,2 \mathrm{ab}$ & $2,29:$ \\
\hline & 3 & 86 & 330 & 3,87 & 29,9 & 10,9 & 1,06 & 403 & 35 & 122 & 169 & 68 & 32,3 & 35,7 & 170 & 13,9 & 2,22 \\
\hline & 6 & 103 & 409 & 4,02 & 29,2 & 8,2 & 1,05 & 425 & 40 & 123 & 178 & 76 & 36,8 & 37,3 & 156 & 14,0 & 2,19 \\
\hline & 9 & 113 & 478 & 4,29 & 32,5 & 7,7 & 1,06 & 456 & 44 & 129 & 198 & 88 & 36,3 & 33,3 & 233 & 14,8 & 2,46 \\
\hline \multirow[t]{4}{*}{ Kenor que 400} & & $114 \mathrm{a}$ & $524 a$ & $4,59 a$ & $37,5 a$ & $\underline{9,6 a}$ & $1,04 a$ & $461 \mathrm{a}$ & $43 \mathrm{a}$ & $126 a$ & $189 a$ & $87 \mathrm{a}$ & $35,8 a$ & $38,4 a$ & $220 a$ & $15,5 \mathrm{a}$ & $\underline{2,38 a}$ \\
\hline & 3 & 92 & 359 & $3,89^{\circ}$ & 40,2 & 14,5 & 1,03 & 436 & 40 & 126 & 180 & 73 & 34,4 & 38,1 & 221 & 15,1 & 2,28 \\
\hline & 6 & 125 & .550 & 4,48 & 39,5 & 9,3 & 1,05 & 466 & 45 & 122 & 183 & 91 & 35,6 & 39,6 & 207 & 15,5 & 2,24 \\
\hline & 9 & 124 & 663 & 5,41 & 32,9 & 5,0 & 1,05 & 481 & 44 & .131 & 205 & 98 & 37,4 & 37,4 & 233 & 15,8 & 2,61 \\
\hline c.v. (x) & . & 23 & 33 & 18 & 13 & 30 & 5 & 10 & 15 & 17 & 10 & 8 & 12 & 10 & 13 & & \\
\hline D.M.S. (57) &. & 42 & 250 & 1,22 & 6,9 & 1,5 & 0,06 & 70 & 10 & 34 & 31 & 10 & 7,1 & 6,5 & 40 & & \\
\hline
\end{tabular}

(1) Os números com a mesma letra não diferem entre si pelo teste de Tukey a $5 \%$.

1/ nmoles $\mathrm{C} 2 \mathrm{H} 4 / \mathrm{vaso} / \mathrm{h}$

2/ nmoles $\mathrm{C} 2 \mathrm{H} 4 / \mathrm{mg}$ núdul $\mathrm{s} / \mathrm{h}$

3/ ARN (Atividade da redutase do nitrato - nmoles NO 2 /g mat. verde/h) 


\section{CONCLUSÕES}

As atividades enzimáticas não sofreram influência pronunciada da calagem, talvez devido à época em que se procedeu à colheita (início da floração), ou ao acúmulo de $\mathrm{N}^{-\mathrm{NH}_{4}^{+}}$no solo, promovendo inibiçōes na atividade da nitrogenase e da redutase do nitrato.

As fontes e doses de calcário não mostraram efeito no nitrogênio amoniacal e nítrico, na época da colheita, a maior parte do nitrogênio mineral provavelmente tendo sido metabolizado antes da floração.

O nitrogênio protéico foi influenciado pela calagem, e o calcário cambucá promoveu os maiores teores dessa forma de nitrogênio, mostrando a influência do magnésio.

A calagem aumentou a absorção dos nutrientes nitrogênio, fósforo, cálcio e magnésio e, em todos os casos, ficou provado o efeito do magnésio, tendo em vista que a maior absorção ocorreu sempre com os calcários com maiores teores desse nutriente.

\section{LITERATURA CITADA}

BRASIL. Levantamento Exploratório - reconhecimento de solos do Estado de Pernambuco. Recife: EMBRAPA, Centro de Pesquisas Pedológicas, 1973. 359p. (Boletim técnico, 26).

BREMNER, J.M. Total nitrogen. In: BLACK, C.A. Methods of soil analysis. Part II. Madison: American Society of Agronomy, p. 1149-1176, 1965.

\& KEENEY, D.R. Stearn distillation methods of determination of ammonium, nitrate and nitrite. Anal. Chem. Acta, Amsterdam, v.32, p. 1149-1176, 1965.

FRANÇA, G.E. de, BAHIA FILHO, A.F.C., CARVALHO, M.M. Influência de magnésio, micronutrientes e calagem no desenvolvimento e fixação simbólica de nitrogênio na soja perene var. Tinarco Glycine wightii em solo de cerrado. Pesq. agropec. bras., Brasília, v.8, n.8, p. 197-202, 1973.

FRANCO, A.A. \& DAY, J.M. Effects of lime and molybdenum on nodulation and nitrogen fixation of Phaseolus vulgaris $L$. in acid soils of Brazil. Turrialba, Costa Rica, v.30, n.1, p. 99-105, 1980.

JONES, J.B. \& FREITAS, L.M.M. de. Respostas de quatro leguminosas tropicais a fósforo, potássio e calcário num Latossolo VermeIho Amarelo de campo cerrado. Pesq. agropec. bras., Brasília, v.5, p. $91-99,1970$.

KEYSER, H.H. \& MUNNS, D.N. Tolerance of rhizobia to acidity, aluminum and phosphate. Soil Sci. Soc. Am. J., Madison, v.43, n.4, p. 519-523, 1979. 
LOVADINI, L.A.C., BULISANI, E.A., MASCARENHAS, H.A.A. Efeito de níveis de calagem, fósforo e potássio na produção de matéria seca de soja perene Glycine wightii, em solos de cerrado. R. bras. Ci. Solo, Campinas, v.1, n.1, p. 31-34, 1977.

MARCUS-WYNER, L. \& RAINS, D.W. Simultaneous measurement of $\mathrm{NH} \dot{4}$ absorption and $\mathrm{N} 2$ fixation by Glycine max L. PI. Physiol., Bethesda, v. 69, n., p. 460-464, 1982.

MORRIS, D.O. The intelligent use of inoculants and lime pelleting for tropical legumes. Trop. Grassl., Oxford, v.1, p. 107-121, 1967.

PURCINO, H.M.A., PURCINO, A.A.C., LYND, J.Q. Efeitos da fertilidade do solo comandando o crescimento e parámetros nodulares à ântese de duas cultivares de feijão-alado num Latossolo Vermelho Escuro (Entrustox) de Minas Gerais, Brasil, Pesq. agropec. bras., Brasilia, v.16, n.5, p. 633-644, 1981.

RAIJ, B. van. O calcário e a calagem. In: UNIVERSIDADE FEDERAL DE GOIÁS. Escola de Agronomia e Veterinária. Curso de Atualização em Fertilidade do Solo. Goiás: 1980. s.p.

RIOS, V., MARTINI, J.A., TEIXEIRA, R. Efect of liming on acidity and contents of extractable aluminum and iron in Mine Panama soils. Turrialba, Costa Rica, v.18, n.2, p. 139-146, 1968.

SANTOS, T.M.B.R. dos. Efeito de alguns nutrientes no metabolismo nitrogenado de Vigna unguiculata (L.) Walp. Recife: Universidade Federal Rural de Pernambuco, 1982. 101 p. (Dissertação de mestrado)

SARRUGE, J.R. \& HAAG, H.P. Análise químicas em plantas. Piracicaba: ESALQ, 1974. 56p.

SCHOLS, D., KOLLING, J., FREIRE, J.R.J. Necessidade de inoculação e aplicação de calcário em leguminosas forrageira tropicais em solos ácidos. $\mathbf{R}_{\mathbf{a}}$ bras. $\mathbf{C l}$. Solo, Campinas, v.5, n.2, p.97-102,1981.

STAMFORD, N.P., NEPTUNE, A.M.L., SILVA, I.P. Efeito do potássio em presença de $\mathrm{N}$-meneral na nodulação, crescimento e absorção de nutrientes por Vigna unguiculata (L.) Walp. R. bras. Ci. Solo, Campinas v.4, n.2, p. 99-103, 1980. 